\title{
Building up hopes
}

As we collectively welcome the new year, we retrace the 2020 milestones in sample return and look at a few of the major upcoming events in 2021. There are many reasons for astronomers to be optimistic.

A t last: 2021. Such anticipation simply for not being 2020! Of course, COVID-19 is still running its course, but with the rollout of several effective vaccines starting in late 2020 and early 2021 , hopes are high for a return to social interactions and the rebuilding of economies within four to six months. Yet throughout the pandemic, dedicated teams have kept space missions going and even launched new missions successfully. There is plenty to look forward to in the coming year.

But first, a quick return to 5 December 2020, when a capsule containing samples from the Hayabusa 2 mission to near-Earth asteroid (162173) Ryugu touched down on Earth. The samples were collected both from the surface and from a newly created crater made by an impactor fired from the spacecraft. The latter will include pristine material, without the weathering and impacts of bodies that alter the properties at the surface. Having both surface and subsurface samples will allow researchers to gain insight into the role that carbonaceous asteroids such as Ryugu had in the delivery of water and organic matter to Earth.

The capsule from Hayabusa2 has already been opened and its contents will be analysed in the next few months. The equivalent samples from Bennu should arrive back on Earth in 2023 after being collected and stowed safely in October 2020. The sample from Bennu, probably well in excess of $60 \mathrm{~g}$, is significantly larger than that from Ryugu $(\sim 5 \mathrm{~g})$. Browse our Collection of papers from the OSIRIS-REx mission's approach to Bennu, which includes the Editorial where we discuss the importance of sample return for planetary research. Meanwhile, important information can be retrieved by analysing the in situ observations. Two papers in this issue show bright boulders, on both Bennu and Ryugu, that point to the formation history of the asteroids, as explained in the accompanying News \& Views.

The Mars Sample Return campaign, a NASA-ESA collaboration, is also well underway. Launched in July 2020, NASA's Mars 2020 Perseverance rover will arrive at Mars in February 2021 and start drilling at selected sites, collecting rocks and soil samples. These samples will be cached for later retrieval and delivery by a 'fetch rover' to an ascent vehicle, which will rendezvous with an orbiter and send them back to Earth, in 2031 at the earliest, where they will be analysed for - among other things - evidence of past life. Data gathered in situ by the mission will also inform the development of potential crewed missions to the Red Planet. Although this plan sounds very ambitious, a recent Chinese mission has demonstrated a scaled-down version admirably.

On 1 December 2020, the Chinese space agency's Chang'e 5 mission touched down on the Moon and returned a sizeable sample of lunar rocks and soil to Earth on 16 December. Compared with Apollo mission samples - estimated to be between 3.1 and 4.4 billion years old - Chang'e 5 's landing zone (in the northwest area of the Moon's near side) contains younger geological structures closer to 1.2 billion years old, which will be useful for a better calibration of the ages of other Solar System features. It is also the first batch of lunar samples returned to Earth since 1976. Future missions Chang'e 6, 7 and 8 have already been planned within the next decade, in preparation for a crewed mission. Surely it has been a record year for sample return!

But there's more in the works for 2021 beyond planetary science missions. Largely due to the pandemic, the 2020 campaign of the Event Horizon Telescope did not take place. Instead, the team has been hard at work preparing for the March 2021 observations, to which they have added two new telescope nodes: the Northern Extended Millimeter Array in the French Alps and the Kitt Peak telescope in Arizona, United States. With a total of eleven observatories around the world, more stunning images of black hole shadows are sure to come.

Construction of the Vera C. Rubin Observatory was also affected by the pandemic. After a six-month hiatus, work resumed at the summit of Cerro Pachón, Chile. With the Legacy Survey of Space and Time commissioning camera now on site, the refrigeration system can undergo tests ahead of the camera installation. First light had been planned for October 2021 but that will now be somewhat delayed. Once completed, the ten-year survey of the night sky will provide tracking for billions of objects, taking time-domain astronomy to unprecedented levels.

And last but not least: 2021 really could be the year of the James Webb Space Telescope (JWST), now expected to launch on 31 October. As the successor to the Hubble Space Telescope, the anticipation is off the scale. Recently, the Cycle 1 round of proposals attracted at least 1,170 General Observer proposals competing for a total of 6,000 hours of observing time accompanied by an endless string of memes on Twitter. Because of its infrared sensitivity, JWST is also a natural successor to the Spitzer Space Telescope. It will similarly be able to see through dust and gas, but JWST will be able to see farther into the past, to older and dimmer objects going back to the first stars and galaxies. It was designed to probe the formation and evolution of stars and galaxies, as well as of planets of the Solar System and of other star systems.

Planning further ahead, Astro2020 - the US National Academies report on the Astronomy and Astrophysics Decadal Survey for developing a strategy for frontier astronomy for the next decade - will be published in March 2021. Astro2020 is the first decadal to introduce a Panel on the State of the Profession and Societal Impacts. The panel will provide a health check of the community and establish goals for what the profession should look like in 2030, in terms of "demographics, diversity and inclusion, workplace climate, workforce development, education, and public engagement".

We can hope that this is a sign that the community, as we slowly recover from COVID-19, will aim at rebuilding a better and more inclusive and equitable environment.

Published online: 14 January 2021 https://doi.org/10.1038/s41550-020-01298-5 